\title{
REFLEXIVE BANACH ALGEBRAS
}

\section{E. R. KEOWN}

1. This paper contains a generalization in the commutative case of the structure theorem of $\mathrm{W}$. Ambrose for $H^{*}$-algebras. An immediate result of Ambrose's axioms is that the orthogonal complement of an ideal is an ideal of the same nature. This fact suggests thinking in terms of a Banach algebra whose conjugate space is a Banach algebra such that the annihilator of an ideal in one algebra is an ideal in the other algebra. This is the essential idea in the definition of a commutative, semi-simple, reflexive Banach algebra given in $\$ 2$. This section shows that such an algebra is essentially an $l_{p}$-space in which a product has been defined by means of co-ordinate multiplication. $\$ 3$ contains a few remarks and examples concerning the author's conjecture that a similar structure theorem must exist in the noncommutative case.

The term annihilator will be constantly needed in both its ring and vector space sense. The phrase ring annihilator will be used to distinguish the use of the term in the ring sense from its use in the vector space sense. $B^{\prime}$ will be used to denote the conjugate space of the given algebra $B$. Greek letters $\epsilon, \delta$, and $\rho$ will be used to denote complex numbers. Elements in $B$ will be denoted by letters $x, y$; and $z$, while elements in $B^{\prime}$ will be denoted by $f, g$, and $h$. The value of the linear functional $f$ at the point $x$ will be denoted by $(x, f)$. Projections in $B$ will be denoted by means of the letter $E$, while those in $B^{\prime}$ will be denoted by means of $E^{\prime}$. The following theorems from the general theory will be used.

Theorem (A). The maximal, regular ideals of a commutative Banach algebra are of deficiency one.

TheOREM (B). The Jacobson radical of a Banach algebra is the intersection of all the maximal, regular, right ideals.

2. Definition 2.1. A commutative, semi-simple Banach algebra $B$ will be called reflexive if it satisfies the following conditions.

(a) The elements of $B$ are the points of a reflexive Banach space.

(b) The conjugate space $B^{\prime}$ is a commutative, semi-simple Banach algebra.

(c) The algebraic and metric properties are connected by the following relations. If $I$ is an ideal of $B$, then $A^{\prime}$, the annihilator of $I$, is an ideal of $B^{\prime}$. If $B$ is the direct sum of the ideals $I_{1}$ and $I_{2}$ and if $x_{i}=y_{i}+z_{i}$ with

Received by the editors October 20, 1953 and, in revised form, May 13, 1954. 
$y_{i} \in I_{1}$ and $z_{i} \in I_{2}(i=1,2)$, then $\left\|y_{1}\right\|=\left\|y_{2}\right\|$ and $\left\|z_{1}\right\|=\left\|z_{2}\right\|$ imply $\left\|x_{1}\right\|=\left\|x_{2}\right\|$.

(d) $B$ is related to $B^{\prime}$ in the same manner that $B^{\prime}$ is related to $B$.

A commutative, semi-simple, reflexive Banach algebra will be called a cssr Banach algebra in the remainder of this paper.

Lemma 2.1. If $M$ is a maximal, regular ideal of a cssr Banach algebra $B$, then $B$ is the direct sum of $M$ and a minimal ideal $A$ where $A$ consists of the scalar multiples of an idempotent $E$.

$M$ is known by the general theory of commutative Banach algebras to be of deficiency one. The annihilator $A^{\prime}$ of $M$ must therefore be one-dimensional, i.e. $A^{\prime}$ is a one-dimensional ideal of $B^{\prime}$ and consequently must be the scalar multiples of an idempotent $E^{\prime} . B^{\prime}$ is the direct sum of $A^{\prime}$ and $M^{\prime}$, the ring annihilator of $E^{\prime}$, i.e.

$$
B^{\prime}=M^{\prime}+A^{\prime} \text {. }
$$

This decomposition implies that $B$ is the direct sum of $M$, the null space of $A^{\prime}$, and $A$, the null space of $M^{\prime}$. Since $M^{\prime}$ is a maximal, regular ideal, it follows that $A$ is a one-dimensional ideal, i.e. $A$ must be the scalar multiples of an idempotent $E$.

Lemma 2.2. If $E$ is the idempotent generator of a minimal ideal $A$ of $B$, then there exists a minimal ideal $A^{\prime}$ of $B^{\prime}$ whose idempotent generator $E^{\prime}$ acts as the adjoint of $E$. In other words, if $T_{E}$ is the linear transformation on $B$ whose value at $x$ is $T_{E} x=E x$ and $T_{E^{\prime}}$ is the linear transformation on $B^{\prime}$ whose value at $f$ is $T_{E^{\prime}} f=E^{\prime} f$, then $T_{E^{\prime}}$ is the adjoint transformation of $T_{E}$.

Let $M$ be the ideal which is the ring annihilator of $E$, then $B$ $=M+A . M$ contains every element of the form $x-E x$ so that $M$ is a maximal, regular ideal. The annihilator $A^{\prime}$ of $M$ is the scalar multiples of an idempotent $E^{\prime}$. We shall show that $E^{\prime}$ is the "adjoint" of $E$. We have the two direct decompositions:

$$
B=M+A, \quad B^{\prime}=M^{\prime}+A^{\prime} .
$$

Let $x=x_{1}+x_{2}$ and $f=f_{1}+f_{2}$ with $x_{1} \in M, x_{2} \in A, f_{1} \in M^{\prime}$, and $f_{2} \in A^{\prime}$. Then

$$
(E x, f)=\left(x_{2}, f\right)=\left(x_{2}, E^{\prime} f\right)=\left(x, E^{\prime} f\right) .
$$

The notation of the two previous lemmas will be adopted as standard. Each maximal, regular ideal $M$ of $B$ produces a decomposition of $B$ and $B^{\prime}$ into

$$
B=M+A, \quad B^{\prime}=M^{\prime}+A^{\prime}
$$


where $A$ and $A^{\prime}$ are minimal ideals of $B$ and $B^{\prime}$ respectively. The idempotent generators of $A$ and $A^{\prime}$ are $E$ and $E^{\prime}$ which are the adjoints of each other when considered as bounded, linear operators over their respective algebras. The elements $E$ and $E^{\prime}$ will be referred to as the idempotents related to $M$ and $M^{\prime}$ respectively.

Every maximal, regular ideal $M$ of $B$ generates a multiplicative, linear functional over $B$. If $E$ is related to $M$, then the multiplicative linear functional generated by $M$ must be of the form $g^{\prime}=m E^{\prime}$ for some complex number $m$. Since $g^{\prime}$ is multiplicative, it follows that $\left(E, g^{\prime}\right)=\left(E, g^{\prime}\right)^{2}$ so that $\left(E, g^{\prime}\right)$ is either 1 or 0 . If $\left(E, g^{\prime}\right)$ were 0 , then $g^{\prime}$ would be the zero functional so that $\left(E, g^{\prime}\right)=1 . g^{\prime}$ will be called the multiplicative functional related to $M$. The multiplicative functional related to $M^{\prime}$ is $g$, the "adjoint" of $g^{\prime}$.

Lemma 2.3. If $B$ is a cssr Banach algebra whose underlying space is separable, then $B$ contains only a countable set $\left(M_{i}\right)$ of maximal regular ideals. If $E_{i}$ and $g_{i}^{\prime}$ are the idempotent and multiplicative linear functional related to $M_{i}$, then the set $\left\{E_{i}, g_{i}^{\prime}\right\}(i=1,2, \cdots)$ forms a biorthogonal sequence.

If $M_{1}$ and $M_{2}$ are distinct, maximal, regular ideals with $E_{1}$ and $E_{2}$ their related idempotents, then $E_{1} E_{2}$ belongs to distinct minimal ideals and so is 0 .

$$
\left(E_{1}, g_{2}^{\prime}\right)=\left(E_{1} E_{2}^{\prime}, g_{2}^{\prime}\right)=\left(E_{1} E_{2}, g_{2}^{\prime}\right)=0 .
$$

Thus the idempotent related to one maximal, regular ideal belongs to the null space of the multiplicative, linear functional related to any other maximal ideal. To every multiplicative, linear functional $g^{\prime} \in B^{\prime}$, there corresponds an $x \in B$ of norm one such that $\left(x, g^{\prime}\right)$ $=\left\|g^{\prime}\right\|$. Thus we have the relation $\left\|g^{\prime}\right\|^{2}=\left(x^{2}, g^{\prime}\right) \leqq\left\|x^{2}\right\|\left\|g^{\prime}\right\| \leqq\left\|g^{\prime}\right\|$ so that $\left\|g^{\prime}\right\| \leqq 1$. It is known that in a separable space such a biorthogonal sequence must be countable.

Notice that if $g^{\prime}=m E^{\prime}$ is the multiplicative, linear functional related to $M$, then $g=m E$ is the multiplicative, linear functional related to $M^{\prime}$.

Lemma 2.4. Let $\left(M_{i}\right)(i=1, \cdots, n)$ be a finite set of distinct, maximal, regular ideals of $B$ with $\left(E_{i}\right)$ and $\left(g_{i}^{\prime}\right)$ the set of related idempotents and multiplicative, linear functionals. Let $m_{n}=\cap M_{i}$ and $E$ $=E_{1}+\cdots+E_{n}$ with $I_{n}=B E$. Then $B$ is the direct sum

$$
B=m_{n}+I_{n}
$$

and $B^{\prime}$ is the direct sum 


$$
B^{\prime}=m_{n}^{\prime}+I_{n}^{\prime},
$$

with $m_{n}^{\prime}$ the annihilator of $I_{n}$ and $I_{n}^{\prime}$ the annihilator of $m_{n}$.

Let $A$ be the ring annihilator of $E$. Then

$$
B=A+I_{n}, \quad B^{\prime}=A^{\prime}+I_{n}^{\prime},
$$

with $I_{n}^{\prime}$ the annihilator of $A$ and $A^{\prime}$ the annihilator of $I_{n}^{\prime}$. Let $x \in A$ and $h \in B^{\prime}$. Then $\left(E_{i} x, h\right)=\left(x, E_{i}^{\prime} h\right)=0$ for $i=1, \cdots, n$. Thus $E_{i}^{\prime} \in I_{n}^{\prime}$ and consequently $g_{i}^{\prime} \in I_{n}^{\prime}$ for $i=1, \cdots, n$. This means $M_{i} \supset A$ and $\cap M_{i} \supset A$. If $x \in \cap M_{i}$, then $E x=0$, so that $x \in A$. Thus

$$
A=\cap M_{i} \text {. }
$$

Let $E^{\prime}=E_{1}^{\prime}+\cdots+E_{n}^{\prime}$, then for $h \in I_{n}^{\prime}$ and $x \in B$ we have $(x, h)$ $=(E x, h)=\left(x, E^{\prime} h\right)$, or $(x, h-E h)=0$. This means that $h=E^{\prime} h$ and that the idempotents $\left(E_{i}^{\prime}\right)(i=1, \cdots, n)$ form a basis for $I_{n}^{\prime}$.

Lemma 2.5. If I denotes the ideal of $B$ consisting of all finite linear combinations of the minimal idempotents $\left(E_{i}\right)$, then $I$ is dense in $B$.

$I$ contains all the $I_{n}$ of the previous lemma. Let $m^{\prime}$ denote the annihilator of $I$. Note that $m_{n}^{\prime} \supset m^{\prime}$ for every $n$, where $m_{n}^{\prime}$ is the annihilator of $I_{n}$. Now if $x \in m^{\prime}$, then for every $n$, let $g_{n}$ be the multiplicative functional for $M_{n}^{\prime}$.

$$
\left(g_{n}, x\right)=\left(E_{n} g_{n}, x\right)=\left(g_{n}, E_{n}^{\prime} x\right) .
$$

But $x \in m^{\prime}$, so that $x \in m_{n}^{\prime}$ and $E_{n}^{\prime} x=0$. This means that $\left(g_{n}, x\right)=0$ for every $n$, so that $x$ belongs to all the regular maximal ideals of $B^{\prime}$. Since $B^{\prime}$ is semi-simple, $x=0$. This shows that $m^{\prime}$ is the zero ideal and that $I$ must be dense in $B$.

From the general theory of commutative, semi-simple Banach algebras we have two corollaries.

Corollary (A). The ideals with compact support in the structure space are dense in $B$.

CoROllaRY (B). Every proper, closed ideal may be embedded in a maximal, regular ideal.

Lemma 2.6. The set $\left(E_{i}\right)(i=1, \cdots, n, \cdots)$ is a basis for $B$.

Let $I_{n}$ designate any one of the finite-dimensional ideals of Lemma 2.4. If $x$ is any element of $B$ then $x=x_{n}+z$ where $x_{n} \in I_{n}$ and $z \in m_{n}$. There is an element $f \in B^{\prime}$ of norm one such that $\left(x_{n}, f\right)=\left\|x_{n}\right\|$. Thus

$$
(x, f)=\left(x_{n}, f\right)+(z, f)=\left\|x_{n}\right\|+(z, f) .
$$


If $(z, f)=0$, then

$$
\|x\|=\|x\|\|f\| \geqq(x, f)=\left\|x_{n}\right\| .
$$

If $(z, f)=r e^{i \theta}$, consider the element $x^{\prime \prime}=x_{n}+e^{-i \theta} z$. Then $\|x\|$ $=\left\|x^{\prime \prime}\right\|=\left\|x^{\prime \prime}\right\|\|f\| \geqq\left(x^{\prime \prime}, f\right)=\left\|x_{n}\right\|+r$.

Thus in all cases $\|x\|$ is not less than $\left\|x_{n}\right\|$. We now observe that

$$
x_{n}=\sum_{i=1}^{n}\left(x, g_{i}^{\prime}\right) E_{i}
$$

and our condition reads

$$
\left\|\sum_{i=1}^{n}\left(x, g_{i}^{\prime}\right) E_{i}\right\|=\left\|x_{n}\right\| \leqq\|x\| .
$$

This condition is sufficient to insure that the $\left(E_{i}\right)$ form a basis for $B$.

The set $\left(E_{i} /\left\|E_{i}\right\|\right)(i=1, \cdots, n, \cdots)$ will be denoted in the remainder of paper by $\left(n_{i}\right)$. Since we are interested primarily in the infinite-dimensional case, we shall assume in the remainder of the paper that there are an infinite number of elements in this collection.

LEMMA 2.7. Let $x=\epsilon_{1} n_{1}+\epsilon_{2} h$ and $y=\delta_{1} n_{1}+\delta_{2} k$ where $\left|\epsilon_{1}\right|=\left|\delta_{1}\right|$, $\left|\epsilon_{2}\right|=\left|\delta_{2}\right|,\|h\|=\|k\|=1$, and $E_{1} h=E_{1} k=0$. These conditions imply that $\|x\|=\|y\|$.

Consider the decomposition of $B$ given by

$$
B=B E_{1}+I \text {. }
$$

Then $x=\epsilon_{1} n_{1}+\epsilon_{2} h, y=\delta_{1} n_{1}+\delta_{2} k$ are the expansions of $x$ and $y$ in this direct sum. Since $\left\|\epsilon_{1} n_{1}\right\|=\left\|\delta_{1} n_{1}\right\|$ and $\left\|\epsilon_{2} h\right\|=\left\|\delta_{2} k\right\|$, it follows that $\|x\|=\|y\|$.

LemMA 2.8. Let $x=\epsilon_{1} n_{1}+\cdots+\epsilon_{j} n_{j}$ and $y=\delta_{1} n_{1}^{\prime \prime}+\cdots+\delta_{j} n_{j}^{\prime \prime}$ where $\left|\epsilon_{i}\right|=\left|\delta_{i}\right|(i=1, \cdots, j)$, and the $\left(n_{i}^{\prime \prime}\right)(i=1, \cdots, j)$ are distinct elements of the set of all $\left(n_{i}\right)$. Then $\|x\|=\|y\|$.

First consider the special case

$$
x=\epsilon_{1} n_{1}+\cdots+\epsilon_{j} n_{j}
$$

and

$$
y=\delta_{1} n_{1}+\cdots+\delta_{j} n_{m},
$$

with $m \geqq j$. The case for $j=2$ has been proved above. Assume the lemma holds for $j=r$ and consider

$$
x=\epsilon_{1} n_{1}+\cdots+\epsilon_{r} n_{r}+\epsilon_{r+1} n_{r+1}
$$


and

$$
y=\delta_{1} n_{1}+\cdots+\delta_{r} n_{r}+\delta_{r+1} n_{m} .
$$

Let $E=E_{1}+\cdots+E_{r}$, and consider the decomposition

$$
B=E B+I \text {. }
$$

The induction follows as above. In the general case, one chooses a third element $z=\epsilon_{1} n_{1}^{\prime}+\cdots+\epsilon_{j} n_{j}^{\prime}$ where $\left(n_{1}^{\prime}, \cdots, n_{j}^{\prime}\right)$ is a set of distinct elements which contains no element of the sets $\left(n_{1}, \cdots, n_{j}\right)$ and $\left(n_{1}^{\prime \prime}, \cdots, n_{j}^{\prime \prime}\right)$. By successive applications of the special case it may be shown that $\|x\|=\|z\|$ and $\|y\|=\|z\|$.

The next lemma is a very slight modification of a result of $F$. Bohnenblust, but some of the argument is included for completeness

Lemma 2.9. If $x=\epsilon_{1} n_{1}+\cdots+\epsilon_{k} n_{k}$, then there exists $a p \geqq 1$ such that

$$
\|x\|=\left(\left|\epsilon_{1}\right|^{p}+\cdots+\left|\epsilon_{k}\right|^{p}\right)^{1 / p} .
$$

The argument will be carried out for the case where all the $\left(\epsilon_{i}\right)$ $(i=1, \cdots, k)$ are positive. The previous lemma shows that this is not a restriction. Consider the function

$$
N(\epsilon, \delta)=\left\|\epsilon n_{1}+\delta h\right\|,
$$

where $\|h\|=1$ and $E_{1} h=0$. By Lemma $2.7, N(\epsilon, \delta)$ is independent of the choice of $h$, providing $h$ satisfies the given conditions. Note that $N$ satisfies the following relations.

(a) $N(r \epsilon, r \delta)=r N(\epsilon, \delta)$ when $r$ is positive.

Recalling that the elements of $B$ have their bound as linear functionals over $B^{\prime}$ as their norm, it is clear that

$$
N(\epsilon, \delta) \leqq N\left(\epsilon^{\prime \prime}, \delta^{\prime \prime}\right)
$$

whenever $\epsilon$ is not greater than $\epsilon^{\prime \prime}$ and $\delta$ is not greater than $\delta^{\prime \prime}$.

(c) $N(\epsilon, \delta)$ is a symmetric function of $\epsilon$ and $\delta$.

Now define a new function

$$
N^{\prime}(\epsilon, \delta, \rho)=\left\|\epsilon n_{1}+\delta n_{2}+\rho n_{3}\right\|,
$$

and note that $N^{\prime}$ is a symmetric function of its variables. Note

$$
N[\epsilon, N(\delta, \rho)]=\left\|\epsilon n_{1}+N(\delta, \rho) \frac{\left(\delta n_{2}+\rho n_{3}\right)}{\left\|\delta n_{2}+\rho n_{3}\right\|}\right\| .
$$

Lemma 2.8 shows that $\left\|\delta n_{2}+\rho n_{3}\right\|=\left\|\delta n_{1}+\rho n_{3}\right\|$, so that $N(\delta, \rho)$ $=\left\|\delta n_{2}+\rho n_{3}\right\|$. Also 


$$
N[\epsilon, N(\delta, \rho)]=\left\|\epsilon n_{1}+\delta n_{2}+\rho n_{3}\right\|=N^{\prime}(\epsilon, \delta, \rho) .
$$

In the same manner, $N[N(\epsilon, \delta), \rho]=N^{\prime}(\epsilon, \delta, \rho)$, so that

(d) $N[N(\epsilon, \delta), \rho]=N[\epsilon, N(\delta, \rho)]$.

Finally,

(e) $N(1,0)=\left\|n_{1}\right\|=1$.

Bohnenblust has shown that a function enjoying properties (a) through (e) must be of the form

$$
N(\epsilon, \delta)=\left(\epsilon^{p}+\delta^{p}\right)^{1 / p}
$$

for some $p(0<p \leqq \infty)$. By induction (employing condition (d) above), it follows for real $\left(\epsilon_{i}\right)$ that

$$
\left\|\epsilon_{1} n_{1}+\cdots+\epsilon_{k} n_{k}\right\|=\left(\epsilon_{1}^{p}+\cdots+\epsilon_{k}^{p}\right)^{1 / p} .
$$

For complex coefficients, Lemma 2.8 shows that

$$
\begin{aligned}
\left\|\epsilon_{1} n_{1}+\cdots+\epsilon_{k} n_{k}\right\| & =\left\|\left|\epsilon_{1}\right| n_{1}+\cdots+\left|\epsilon_{k}\right| n_{k}\right\| \\
& =\left(\left|\epsilon_{1}\right|^{p}+\cdots+\left|\epsilon_{k}\right|^{p}\right)^{1 / p} .
\end{aligned}
$$

Corollary. If $x \in B$ and $x=\sum_{i=1}^{\infty}\left(x, g_{i}^{\prime}\right) E_{i}$, then $\|x\|$ $=\left(\sum_{i=1}^{\infty}\left|\left(x, g_{i}^{\prime}\right)\left\|E_{i}\right\|\right|^{p}\right)^{1 / p}$.

The previous lemmas establish the following theorem.

THEOREM 2.1. If $B$ is a separable, cssr Banach algebra of infinite dimension, then there exists $a p(1<p)$ and a set of constants $\left(d_{i}\right)$ such that $B$ is isomorphic to a space of sequences $L$ such that multiplication in $B$ corresponds to co-ordinate multiplication in L. If $x \sim\left(\epsilon_{i}\right)$, then

$$
\|x\|=\left(\sum_{i=1}^{\infty} d_{i}\left|\epsilon_{i}\right|^{p}\right)^{1 / p}
$$

The index $p$ must not be less than one since a Banach space is convex. It must be greater than one since the space is reflexive. It is clear then the nonseparable case may be handled in an analogous case by means of Zorn's Lemma.

3. The above technique does not extend directly to the noncommutative case. By requiring the underlying space to be a Hilbert space, a different set of axioms may be given for the $H^{*}$-algebras of Ambrose in which the dependence on the spectral theorems for proofs may be completely avoided. The author is encouraged by this fact to believe that a noncommutative structure theorem exists in which an algebra is postulated whose underlying space is a reflexive space, 
but whose conjugate space has multiplication defined only for a dense set of elements. Presumably the desired structure theorem would be that the algebra was the set of all infinite matrices $\left(a_{i j}\right)$ such that

$$
\sum_{i=1, j=1}^{\infty}\left|a_{i j}\right|^{p}<\infty
$$

with $1<p<p /(p-1)$.

The author has the result which might be expected, i.e. finitedimensional matrices along the diagonal in the completely continuous case, but has not obtained the general result as yet.

\section{BiBLIOGRAPHY}

1. W. Ambrose, Structure theorems for a special class of Banach algebras, Trans. Amer. Math. Soc. vol. 57 (1945) pp. 364-386.

2. F. Bohnenblust, $A$ n axiomatic characterization of $L_{\mathbf{p}}$-spaces, Duke Math. J. vol. 6 (1940) pp. 627-640.

3. I. Kaplansky, Dual rings, Ann. of Math. vol. 49 (1948) pp. 689-701.

Agricultural and Mechanical College of Texas 